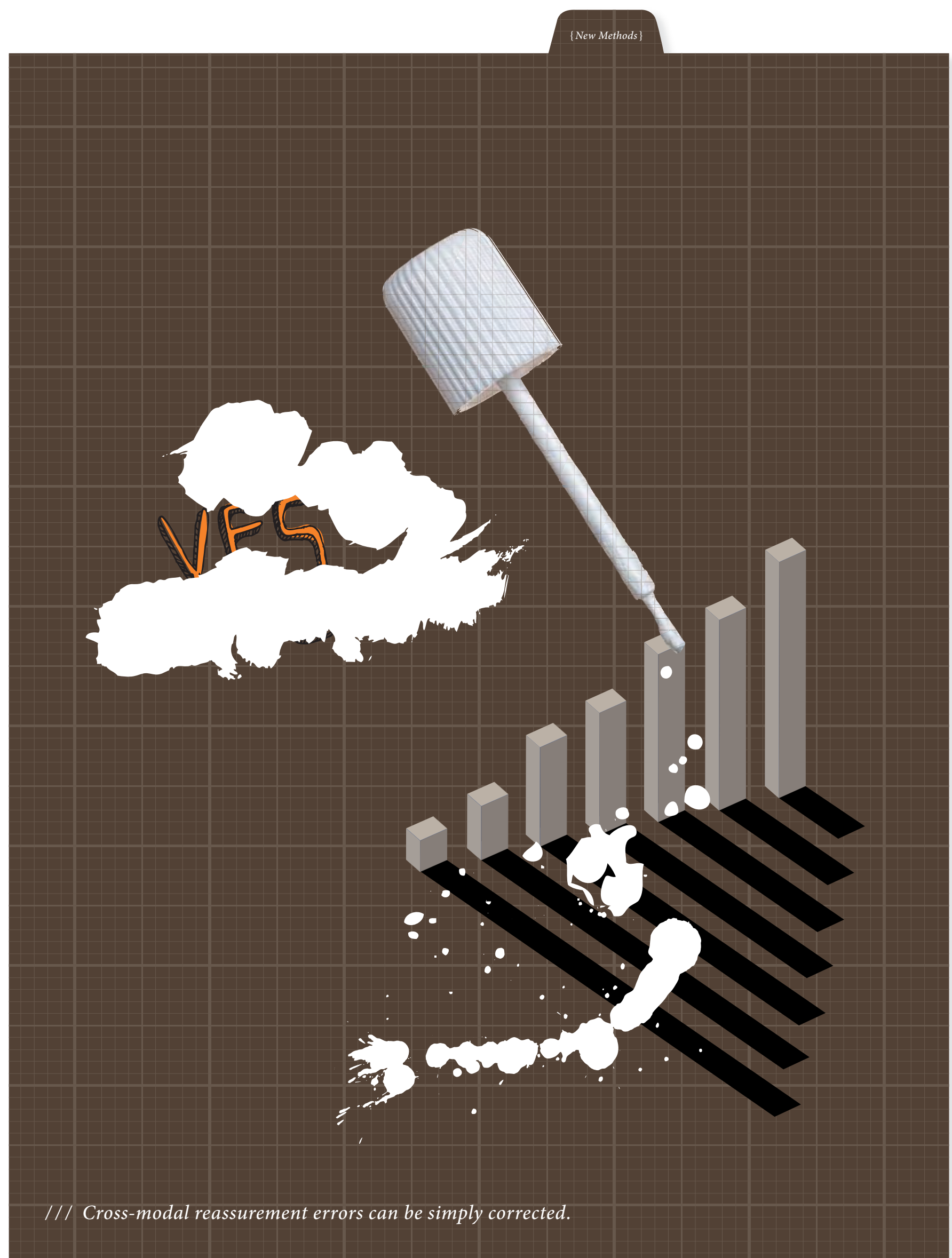




\title{
RESPONSE STYLES AND HOW TO CORRECT THEM
}

\author{
Bert Weijters, Maggie Geuens and Niels Schillewaert
}

Cross-mode surveys are on the rise. Unfortunately, data obtained from different modes of data collection (e.g., telephone and online data) may not be comparable due to measurement bias, especially differences in acquiescence, disacquiescence, extreme and midpoint response styles. This article discusses a study that finds response style differences between data based on the same questionnaire, but obtained by different modes of data collection: paperand-pencil questionnaires, telephone interviews, and online questionnaires. Similar problems may also occur in cross-national data. We propose a new method to measure response styles and correct for them: the representative indicators response style means and covariance structure (RIRSMACS) method.

Cross-mode Surveys: Efficiency vs. Validity

Market research in the past mainly relied on telephone interviews or mail questionnaires, but online surveys have been booming over the last fifteen years. In crossmode surveys, researchers merge data obtained by means of different modes of data-collection, e.g., telephone, paper-and-pencil (P\&P), and online. As they combine the efficiency offered by online surveys with good coverage of the total population of interest (including non-internet users), cross-mode surveys are on the rise.

Unfortunately, the same response to the same question may have a different meaning in a different mode. For example, when using seven-point rating scales, a six may be perceived as a more extreme response online than it is by telephone. Reasons include sensory differences (visual versus auditory presentation), differences in felt time pressure, etc.

To capture such differences in responding and to correct for the resulting bias, market researchers can use the newly developed RIRSMACS method. RIRSMACS stands for Representative Indicators Response Style Means And Covariance Structure. RIRSMACS is a method to include measures of response styles in your questionnaire and data analysis. Response styles are respondents' tendencies to select specific subsets of response options disproportionately in favor of the positive side (acquiescence response style, or ARS, showing a higher selection frequency of 5, 6 and 7 on a 7-point scale, for example), the negative side (disacquiescence response style, or DRS, showing a higher selection frequency of 1,2 and 3 in case of a 7-point scale), the extremes (extreme response style, or ERS, as evidenced by more often selecting 1 or 7 when a 7-point scale is used), or the middle of the scale (midpoint response style, or MRS, meaning more often selecting 4 on a 7 point scale).

The key ingredient of the method is a random sample of marketing items that need to be included in the questionnaire to capture response styles in a reliable and valid manner. Based on these items, the RIRSMACS approach allows for the simultaneous measurement of multiple response styles. This is important because it is difficult to select only those response styles that will matter in advance. The method measures response styles in such a way that measurement error in the response style measures is corrected for (by using a Means And Covariance Structure rather than just using observed scores).

What's the problem? Response styles across modes of data collection

Data analysis methods are becoming more refined, and increasingly sophisticated methods and software packages are becoming available, such as Structural Equation Modeling (cf. the popular and user-friendly AMOS software, for example). Unfortunately, a discrepancy exists between our limited understanding of the response

\section{THE AUTHORS}

B. Weijters

is Assistant Professor of Marketing at Vlerick Leuven Gent Management School, Reep 1, 9000 Ghent, Belgium bert.weijters@vlerick.be

\section{N. Schillewaert}

is Associate Professor of Marketing at Vlerick Leuven Gent Management School, Reep 1, 9000 Ghent, Belgium niels.schillewaert@vlerick.be

M. Geuens

is Professor of Marketing at Vlerick Leuven Gent Management School and Ghent University, Hoveniersberg 24, 9000 Ghent, Belgium maggie.geuens@ugent.be

The article is fully new written based with permission from Journal of the Academy of Marketing Science on: Weijters, Bert, Niels Schillewaert, and Maggie Geuens (2008): Assessing response styles across modes of data collection, Vol. 36, issue 3, pp. 409-422. 
process and our advanced models to represent it. For instance, remarkably little is known on the impact of mode of data collection on response styles. This question is cru-

\section{SIMULATION OF A REALISTIC CROSS-MODE SCENARIO}

A simulation study demonstrated that the RIRSMACS approach is able to identify and correct response style bias in realistic situations where an uncorrected analysis would lead to erroneous conclusions.

The fictitious scenario of the simulation study was the following. A telecom provider has three segments in its database: (1) one segment consists of its telephone customers, (2) a second of its Internet services customers, (3) and the third of combined Internet and telephone customers. The company wants to upgrade the first two segments to combined users by offering a special package of services. An advertisement has been developed to present this package. The company is interested in measuring three focal variables: Attitude toward the $\mathrm{Ad}$ (Aad), Attitude toward the Brand (Ab), and Purchase Intention ( $\mathrm{Pi})$. It was assumed that these variables' averages are located below ( $\mathrm{Pi})$, around $(\mathrm{Ab})$ and above (Aad) the neutral point (the four in a seven-point scale).

The simulation showed that in a realistic and relevant setting using an uncorrected model would lead to false conclusions on group differences. Specifically, the uncorrected model incorrectly suggested significantly higher Aad and Ab levels in the Telephone group. This response style artefact was resolved in the RIRSMACS corrected model. Without correction, a market study might have led to the decision that the Telephone segment would be more easily converted, and the online segment might be underserved as a consequence.

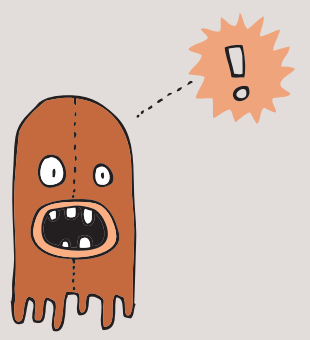

cial as the choice of data collection mode(s) has significant budgetary implications. Market researchers need more information to make optimal decisions in this regard.

Let's take a closer look at some popular modes of data collection: self-administered paper and pencil (P\&P) questionnaires, telephone interviews, and self-administered online questionnaires. The P\&P mode differs from the telephone mode in several important aspects, including the absence/presence of an interviewer. The interviewer's presence may motivate respondents to provide an answer other than the midpoint because respondents feel that would not be satisfactory. The P\&P questionnaires are selfadministered and thus self-paced, but in the telephone mode, an interviewer is largely in control of the process. This can speed up the process to some extent, if only because silences on the phone are experienced as awkward. Time constraints typically lead respondents to respond in a more stylistic way (i.e., more driven by response styles than by content). In sum, there is reason to believe that response styles may be different for the telephone mode and the P\&P mode.

The online mode resembles P\&P in most respects, including visual perception of the questions, manual response to the questions, and self-administration. There seems to be less reason to expect response style differences between the P\&P and the online modes. Note that in the current study we focus on cross-mode differences in response styles, but the correction method we propose can also be applied to cross-national datasets.

\section{THE RIRSMACS METHOD}

\section{Challenges}

Creating useful response style measures is quite challenging for several reasons. First, you need a complete profile of response styles - that is, ARS, DRS, ERS, and MRS. A reduced set may miss important sources of bias.

Second, each response style has to be measured by means of multiple indicators. This is necessary to be able to assess internal consistency (e.g., by means of the Cronbach's alpha coefficient for reliability) and cross-group equivalence. Also, it allows one to account for random 
measurement error, as using response style measures containing measurement error for correction purposes would pass on the random error to the "corrected" measures (which would be rather ironic).

Third, response style measures should be based on a representative sample of heterogeneous items. It may be tempting to use convenience samples of items to measure response styles (i.e., use the items that happen to be in the questionnaire too). The use of a random sample of items is preferable because heterogeneity of the content of the items ensures that the observed response tendencies are not content specific (at which point the tendencies would no longer classify as response styles, as a matter of fact).

In summary, RIRSMACS uses representative samples of items to create multiple indicators of multiple response styles that can be modeled in a Means And Covariance Structure. Exhibit 1 discusses a simulation study that demonstrates how RIRSMACS outperforms alternative methods in correcting for cross-mode response style bias (Refer to Exhibit 1).

Steps to Follow

RIRSMACS consists of the following steps:

1. Collect data on response styles:

a) Set up your cross-mode data collection in such a way that the questionnaires are identical across modes.

b) Randomly sample marketing items from existing scales, using previous questionnaires and/or marketing item inventories. Include the items in your questionnaire in three random blocks, using the same response format as the items you want to correct for response style bias. On the basis of a sensitivity analysis, we suggest that a sample of 6 random items is the absolute minimal requirement to have a workable response style factor model, but a sample of 15 items is recommended. To minimize respondent burden, you can reduce the number of other items in the questionnaire (that measure the variables of interest for your study). It is becoming clear that using many items to measure one construct may induce artificial internal consistency (i.e., increase Cronbach's alpha) without necessarily adding much information. Therefore, it seems recommendable to reduce the number of

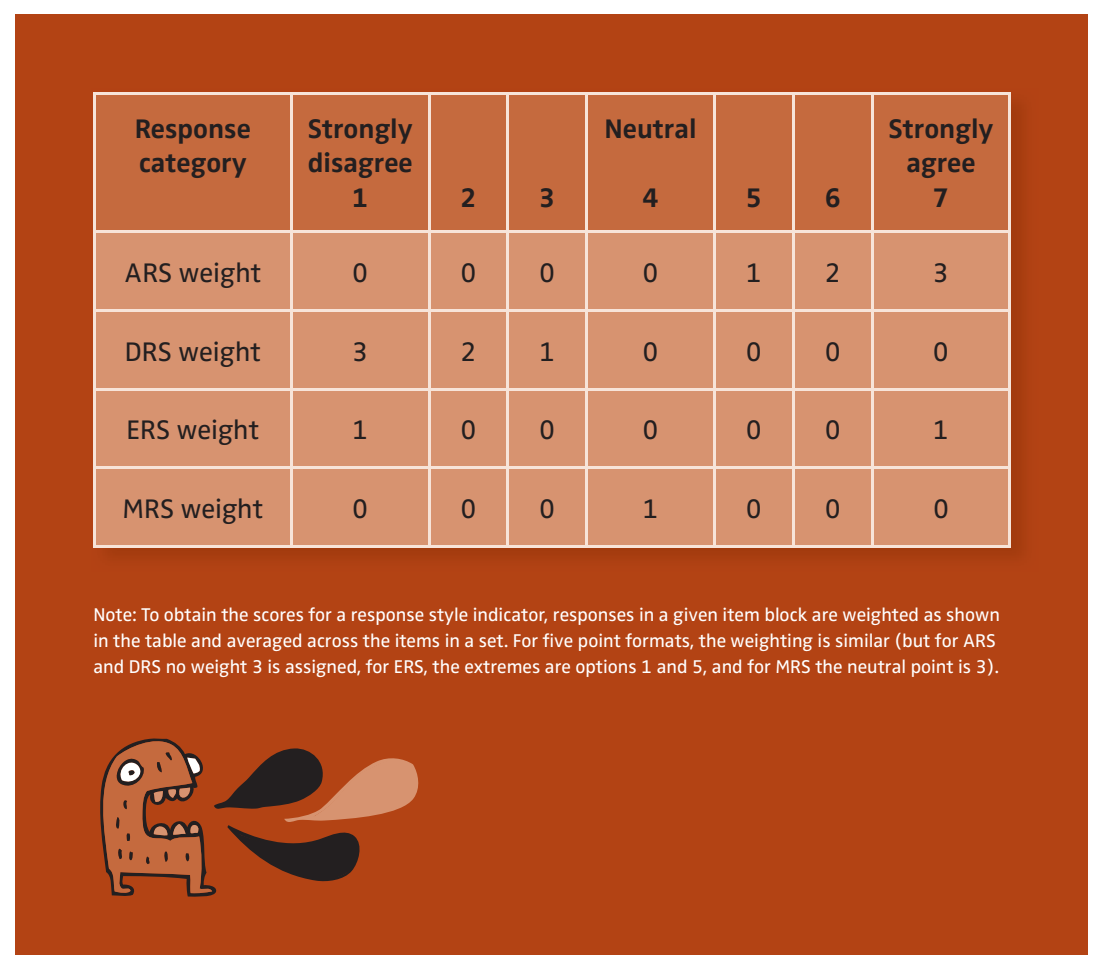

items used to measure constructs and invest the freed questionnaire space in response style measurement. For example, in a study with five constructs, reducing the number of items per scale from six to three would already provide sufficient room for valid response style measurement.

2. Include response styles in your analyses

a) Based on each item block, compute an indicator for ARS, DRS, ERS and MRS using the weights shown in Table 1 to obtain an averaged response style measure per item block and per response style (i.e., each item response is recoded according to the weights in the table and all weights are averaged across the items in one block). The result is 12 indicators, based on three item blocks times four response styles.

b) Create response style factors using the MACS shown in Figure 1, Panel I. This model can be implemented in commonly used software packages for Structural Equation Modeling (e.g., AMOS, Mplus, LISREL, EQS...). Note the specific structure of residual correlations in the model. These correlations represent relations between response style indica-
TABLE 1

Response style indicator coding scheme 




») The same response to the same question may have a different meaning in a different mode. For example, when using seven-point rating scales, a six may be perceived as a more extreme response online than it is by telephone. «

\section{EMPIRICAL STUDY}

Sample and Questionnaire

To compare different modes of data collection, we administered exactly the same questionnaire via three different modes, using samples from the same geographic area: (1) a self-administered P\&P questionnaire, which was recruited by means of a door-to-door random walk procedure $(N=501$; response rate $=58.0 \%$, (2) telephone interviews with a sample taken from the general population ( $\mathrm{N}=496$; response rate $=32.0 \%$ ); and (3) a self-administered online survey with a panel from an online market research company, which was recruited by means of a personalized e-mail $(\mathrm{N}=535$; response rate $48.2 \%$ ). It is important to note at this point that the three samples were balanced for age, gender and educa- tion level (by means of re-sampling). In other words, the P\&P, telephone and online samples had the same profile for these three demographics to ensure that response style differences between the three modes could be attributed to the mode effect and not to demographic effects. (We decided to not use an alternative approach where the same respondents would answer the same questionnaire via different modes, as repeated administration of the same questionnaire would lead to reduced motivation and more fatigue). As a matter of fact, as response styles tend to differ across different socio-demographic segments, it is even useful to implement response style corrections in single-mode data.

The questionnaire consisted of 52 unrelated items from different scales (average inter-item correlation $=0.07$ ), randomly sampled from a compilation of multi-item scales and measured on 7-point agreement rating scales. Note that we use more items than strictly necessary to measure response styles to optimize measurement quality. To assess the impact of response styles on a relevant marketing measure, we included a multi-item measure of trust in frontline employees (TRUST) in a clothing retail context.

Main Findings: Cross-mode Response Style Differences We computed response style indicators based on the random items and applied the model in Figure 1. After testing for measurement equivalence of the response style factors across the modes (i.e., the factor structure was the same), we could compare the latent response style means, as presented in Figure 2. For a good understanding of Figure 2, it is useful to keep in mind the way the response styles are measured. ARS indicates the extent to which respondents tend to lean towards the disagreement side of the rating scale, DRS the extent to which respondents tend to lean towards the negative categories of the rating scale. ARS and DRS are expressed in a scale that reflects the seven-point scale. And, although this may be counter-intuitive, ARS and DRS can occur simultaneously (i.e., in the same respondent), more specifically for respondents who tend to differentiate their responses by avoiding the midpoint. If one subtracts respondents' DRS score from the same respondents' ARS score, the result indicates the net bias away from the midpoint (4 on a seven-point scale). For example, a respondent with an ARS score of 1.5 and a DRS score of 0.5 , will have an expected net bias of 1.5 $0.5=1$; in other words, this respondent is expected to give an average rating of 4 (i.e., the midpoint) + 1 (i.e., ARS-DRS) $=5$ (on a seven-point scale), irrespective of content (Refer to Figure 2). 


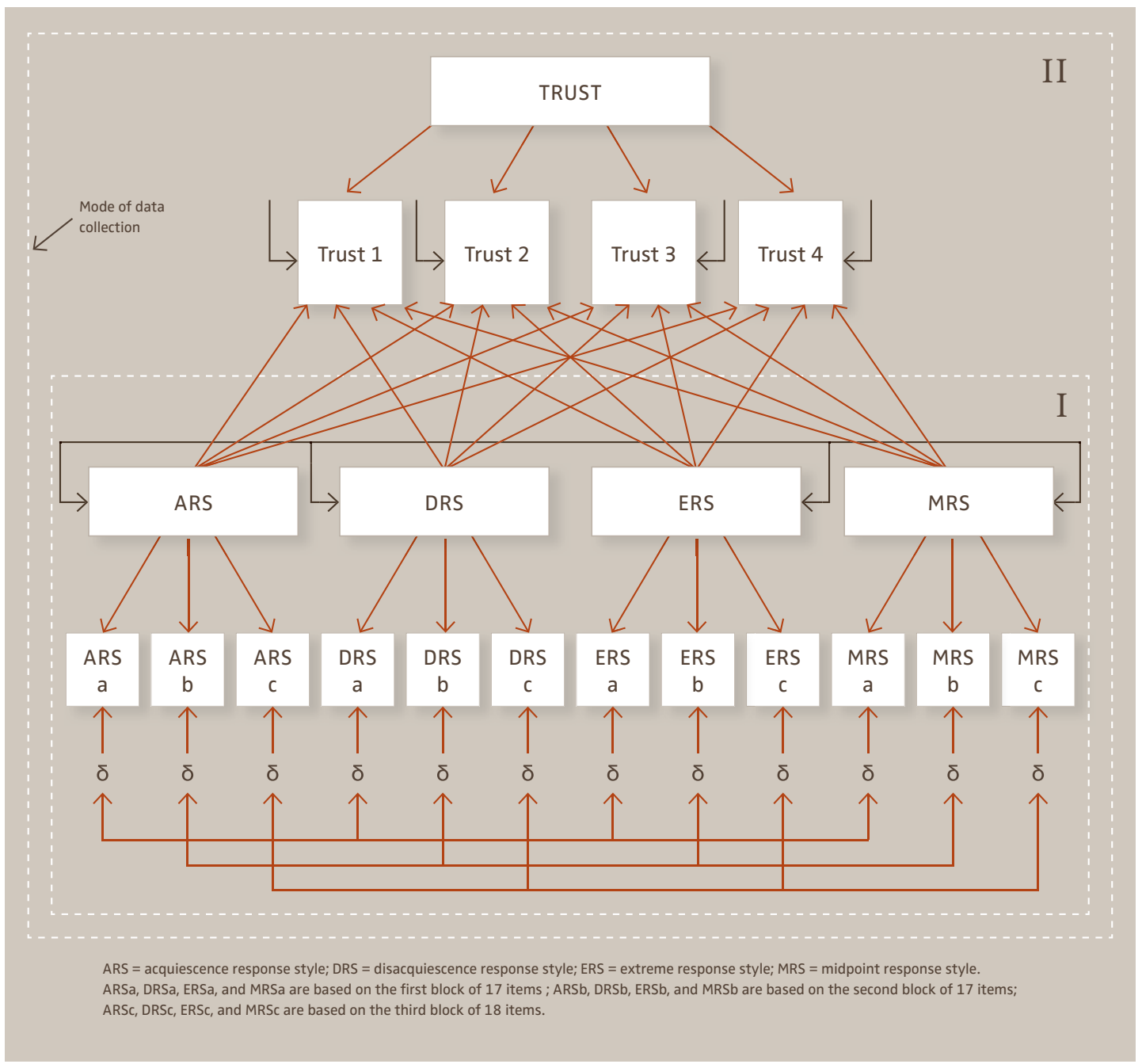

FIGURE 1:

RIRSMACS Model for

Cross-Mode Response Style

Comparison (I) and

Correction (I+ II)
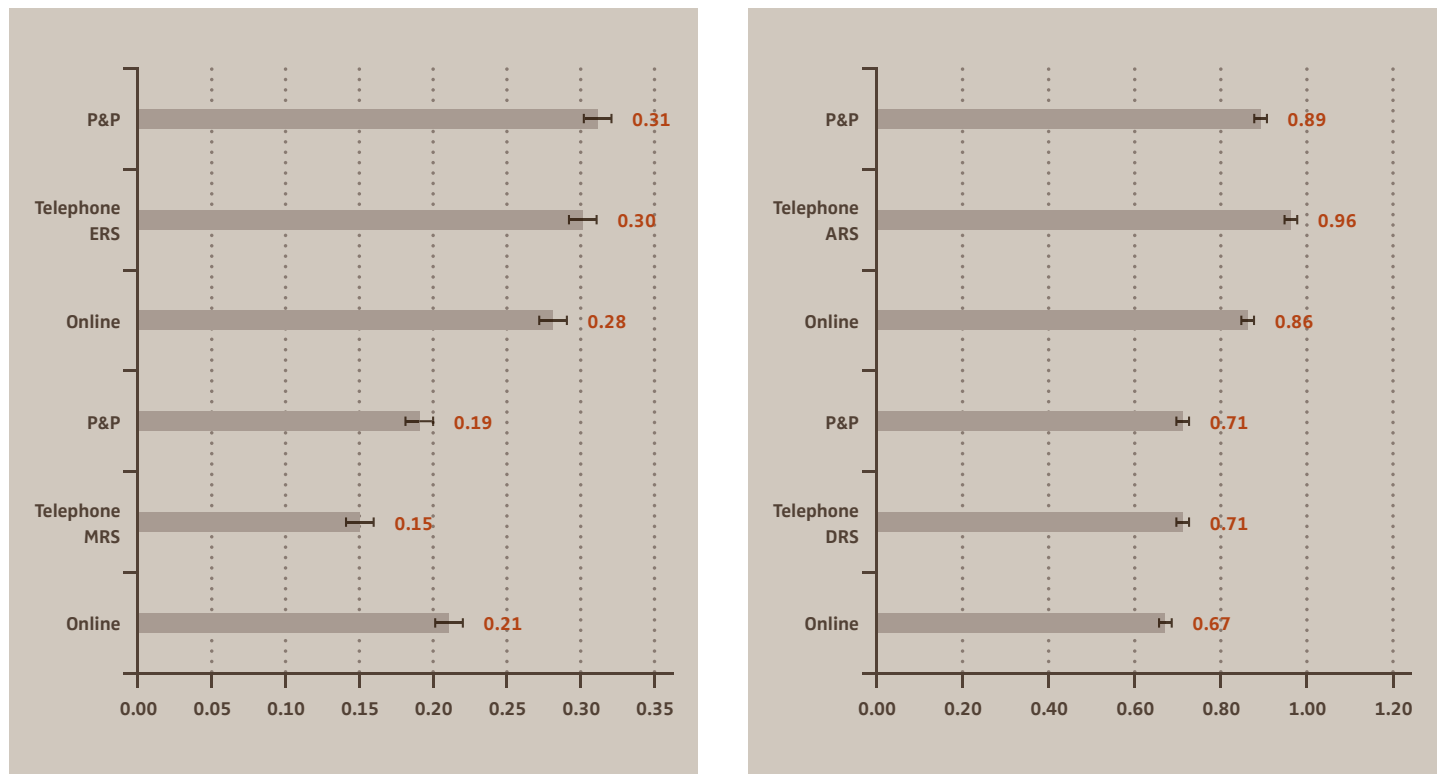

FIGURE 2:

Response style means across modes of data collection

The bars represent the response style means in the different modes and the error bars represent the standard errors of the means.

ARS = acquiescence response style DRS = disacquiescence response style ERS = extreme response style MRS = midpoint response style P\&P = Self-administered Paper \& Pencil data TELE = Telephone survey data Online $=$ Online panel survey data 
Figure 3:

CROSS-MODE FREQUENCY DISTRIBUTIONS (RESPONSE PERCENTAGES) OF TRUST ITEMS

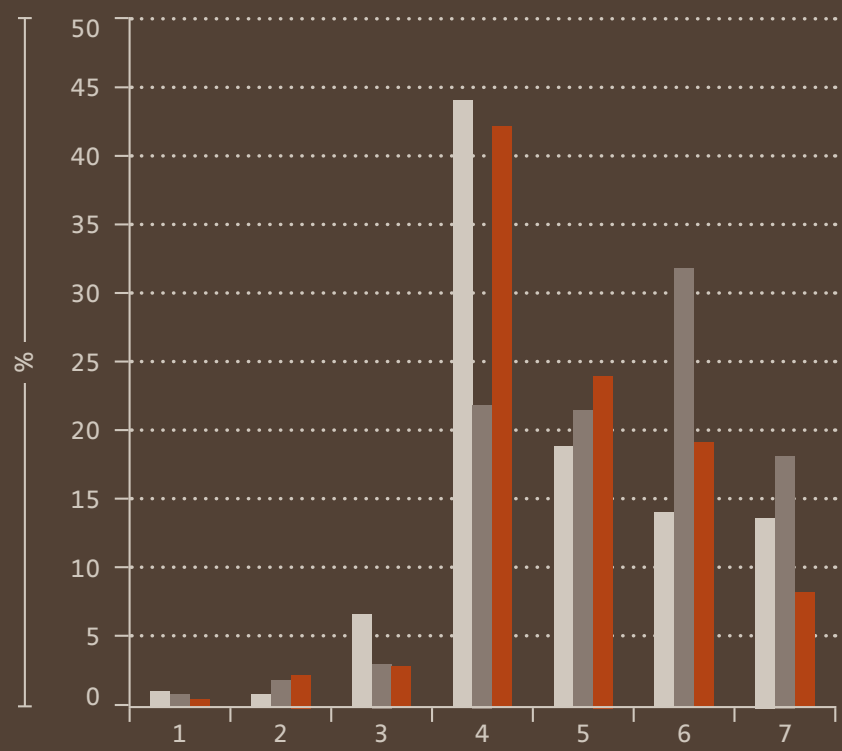

I feel that the employees of this store are very dependable.

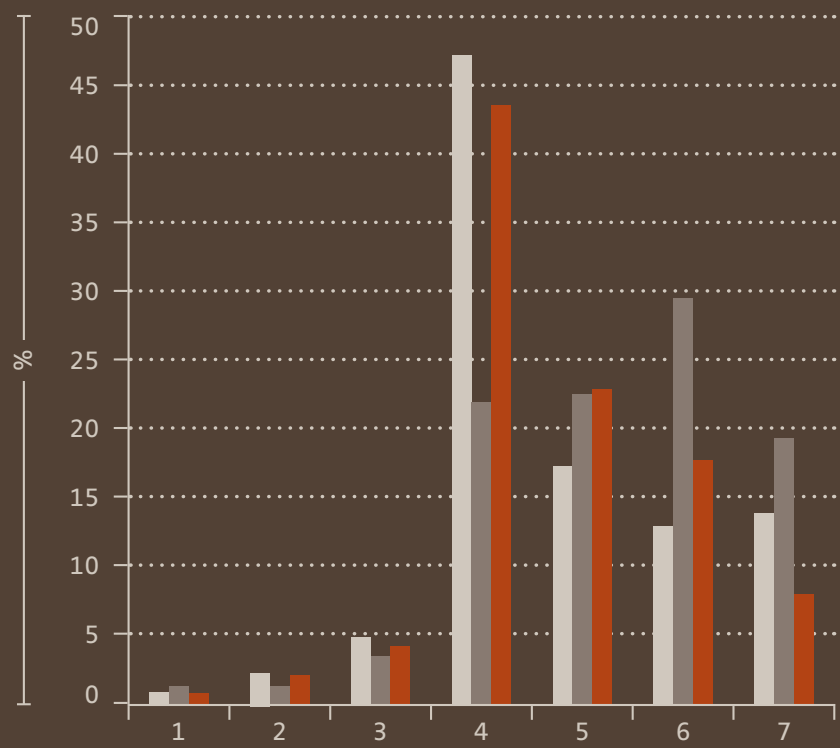

I feel that the employees of this store are of very high integrity.

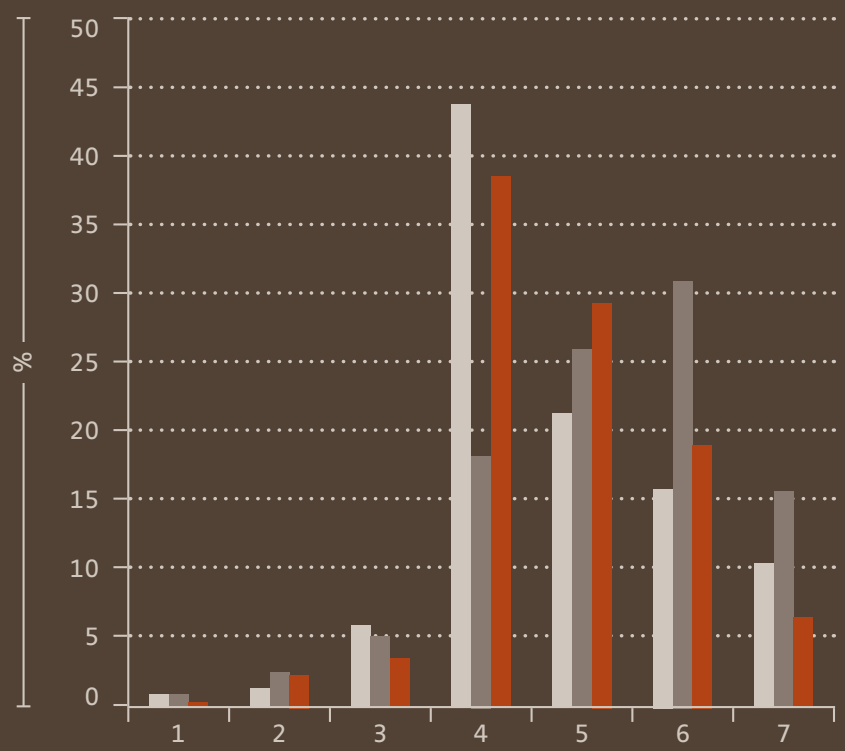

I feel that the employees of this store are very competent.

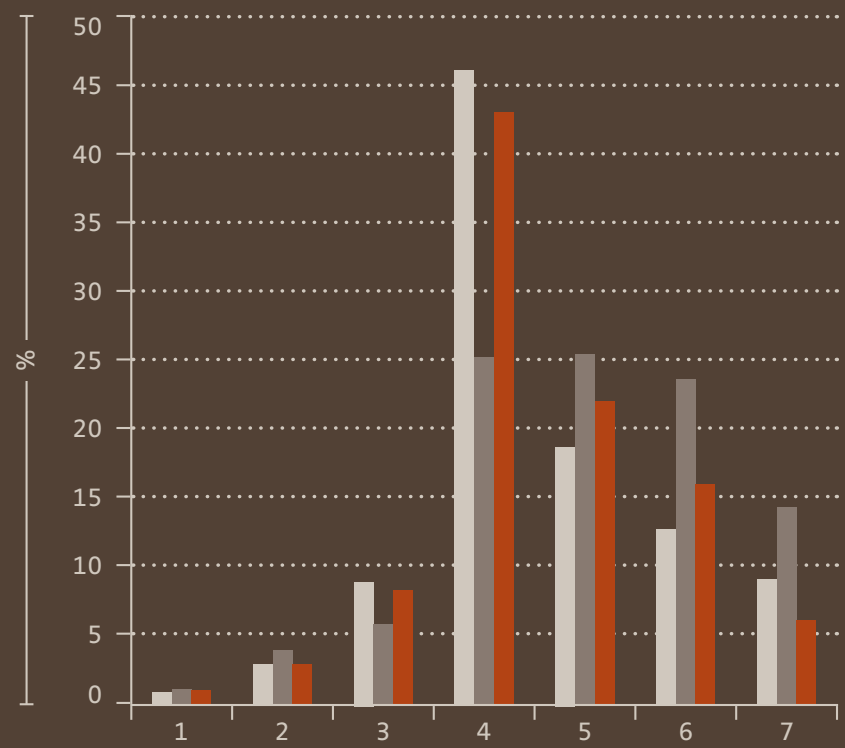

I feel that the employees of this store are very responsive to customers. 
MRS is lower while ARS is higher in the Telephone data. In the telephone group, the probability of respondents choosing the neutral point of a scale (MRS) is markedly smaller than in the other modes. The responses are shifted to the positive side, as reflected by the slightly higher ARS level. Respondents may feel pressure to provide an opinionated response, leading to lower MRS. In addition, the presence of an interviewer might increase the perceived time pressure, thus increasing ARS.

The online data have lower DRS and ERS. The whole response style profile of the online group suggests a moderate way of responding, with the highest MRS and the lowest ARS, DRS, and ERS. Note that the net effect of ARS and DRS leads to a nearly identical expected score for the online and P\&P groups. Conversely, in terms of spread, the expected response distribution for the online group has less heavy tails (as shown by the lower ERS value).

The telephone MRS score of 0.15 indicates that in the telephone mode, on average, $15 \%$ of respondents will select the middle response option in response to a random item, as opposed to $19 \%$ and $21 \%$ in the P\&P and online groups, respectively. In other words, approximately one-fourth of the midpoint responders in the P\&P or online groups might have chosen a different (probably more favorable) option in the telephone mode.

\section{Effect on a Marketing Measure: Trust}

In the current data set, apart from being included in the same questionnaire, the TRUST items were entirely unrelated to the response style measures; the content of the items did not overlap with any of the items in the response style indicators, and we did not use the items themselves to compute the response style indicators. As a result, any relationship between the observed response style levels and the four items can only be attributed to shared response style bias.

The bar charts of the TRUST items in Figure 3 visualize how response style differences among modes may bias cross-mode comparisons of observed scores. As one would expect based on the response style differences shown in Figure 2, the telephone group shows substantially lower frequencies of the middle response and slightly higher frequencies of favorable responses. If the

\section{\) RIRSMACS uses representative samples of items to create multiple indicators of multiple response styles that can be modeled in a Means And Covariance Structure. «}

response style data had not provided clear information on the cross-mode differences in response distributions, the observed scores could easily be ascribed to real content-related differences, and post hoc explanations could probably be provided for the observations.

\section{RIRSMACS to the Rescue}

When applying the RIRSMACS model (see Figure 1), the artificial mean differences (in particular, a significantly higher mean in the telephone data) for the TRUST variable disappear. In particular, in the uncorrected model the factor means for Trust are 4.76, 5.31, and 4.78 for the P\&P, Telephone and Online samples. In the corrected model, the factor means are corrected downwardly, resulting in estimates of $3.30,3.60$, and 3.34 for the P\&P, Telephone and Online samples. Compared to the P\&P mean, the Telephone sample scores significantly higher on Trust in the uncorrected model $(t=6.85, p<0.001)$, but not in the corrected model (1.284; $p>0.10)$.

Bias due to response styles tends to be especially strong and misleading in data collection modes in which the factor structures seem to be highly reliable (largely because of the bias), as was the case in the telephone group. Thus, measures of internal consistency (like Cronbach's alpha) are not sufficient indication of data quality and might even be counterproductive as a criterion to evaluate modes.

The results of the response style mean comparison are important and show that telephone interview data should be handled with caution, in that they may show bias compared with other data. Telephone survey participants tend to use rating scale options away from the midpoint 
» The results of the response style mean comparison are important and show that telephone interview data should be handled with caution, in that they may show bias compared with other data. Telephone survey participants tend to use rating scale options away from the midpoint (mostly positively biased). «

(mostly positively biased). For the online data, we found slightly lower levels of DRS and ERS, which indicates a more moderate way of responding to items.

Finally, the current study shows that cross-mode data may be incomparable without corrective measures. The RIRSMACS approach is the appropriate corrective tool.

RECOMMENDATIONS: WHEN TO DO A RIRSMACS STUDY?

For every study that uses cross-mode data, it is necessary to provide evidence that the modes under comparison are similar in terms of response styles. Given the generalizability of the response style measures in RIRSMACS (the items are a random sample representative of a broader population of questionnaire items), such evidence can be based on available studies that compare the same modes using comparable samples of items and respondents.

A dedicated RIRSMACS study is required under the following conditions: First, in situations in which the crossmode approach is more cost effective (even after including a RIRSMACS study) and is the best guarantee for good population coverage. This may have different reasons. Cross-sectionally, different segments of consumers may be accessible only by means of different modes. For example, a multichannel retailer may want to compare satisfaction and trust between its online customers (using an online questionnaire) and its telephone customers (using telephone interviews). Longitudinally, the cost of different modes of data collection may change to an extent that makes it almost unavoidable to switch modes. For example, a large-scale survey in Western Europe using telephone interviews may be faced with increasing cost and decreasing success of telephone surveying, whereas the Internet is approaching full coverage and is becoming increasingly efficient for surveys.

A second condition is that the modes of data collection should be sufficiently dissimilar to warrant concern about bias due to response styles. For example, this may be the case when telephone and online data are combined.

Third, the questionnaire of interest should not already contain a large enough pool of highly dissimilar items. Such diversity in content is typically lacking in consumer surveys (e.g., in satisfaction surveys) in that variables are usually included in the same questionnaire because they presumably have some relationship.

Finally, a condition for a dedicated study is that no RIRSMACS study is available that assesses the same modes for the same segments in the same language and culture. For the situations that meet these criteria, a RIRSMACS study is required. If no evidence is provided that different modes are similar in terms of response styles, data analysis across modes is non-informative. The reason is that the same response may not have the same meaning in different modes.

In terms of data-analysis (costs), the additional demands of applying the RIRSMACS method is relatively limited on the condition that the analyst uses Structural Equation Modeling already. If this is the case, data preparation takes an extra hour for creating response style measures. SPSS syntax is provided in appendix to this end. For the actual data-analysis, it is likely that approximately half a working day or 4 working hours of a Structural Equation Modeling trained analyst are required for implementation of the response style correction to a single market study using cross-mode data containing several multi-item scales. The benefit is that cross-mode data become interpretable without continuously needing to worry about the possibility of cross-mode response style bias. • 


\section{FURTHER READING}

Baumgartner, Hans, and Jan-Benedict Steenkamp (2006) Response biases in marketing research.

In: Grover, Rajiv, and Marco Vriens (Editors). The handbook of marketing research: Uses, misuses and future advances. Sage Publications.

Deutskens, Elisabeth C., Ko de Ruyter, and Martin G.M. Wetzels (2006)

"An Assessment of Equivalence Between Online and Mail Surveys in Service Research."

Journal of Service Research 8 (4): pp. $346-355$.

Podsakoff, Philip M., Scott B. MacKenzie, Jeong-Yeon Lee, and Nathan P. Podsakoff (2003)

"Common Method Biases in Behavioral Research: A Critical Review of the Literature and Recommended Remedies."

Journal of Applied Psychology 88 (5): pp. 879 - 903.
APPENDIX: SPSS SYNTAX TO COMPUTE RESPONSE STYLE INDICATORS

This is SPSS syntax to compute three indicators (labeled a, b, c) for ARS, DRS, ERS and MRS based on 15 random items (labeled item 1 through item 15).

${ }^{*}$ As a first step, compute auxiliary variables indicating * the frequency of using category 1 'strongly disagree', 2 'disagree', etc. COUNT one_a $=$ item 1 TO item5 (1). COUNT one_b $=$ item6 TO item10 (1). COUNT one_c $=$ item 11 TO item 15 (1). COUNT two_a $=$ item1 TO item5 (2). COUNT two_b $=$ item6 TO item10 (2). COUNT two_c $=$ item11 TO item 15 (2). COUNT three $\_$a $=$item1 TO item5 (3). COUNT three_b $=$ item6 TO item10 (3). COUNT three_c $=$ item 11 TO item15 (3). COUNT four_a $=$ item 1 TO item5 (4). COUNT four_b $=$ item6 TO item 10 (4). COUNT four_c $=$ item11 TO item 15 (4). COUNT five_a $=$ item 1 TO item5 (5). COUNT five_b $=$ item6 TO item10 (5). COUNT five_c $=$ item 11 TO item 15 (5). COUNT six_a $=$ item1 TO item5 (6). COUNT six_b $=$ item6 TO item10 (6). COUNT six_c $=$ item11 TO item15 (6). COUNT seven_a $=$ item 1 TO item5 (7). COUNT seven_b $=$ item6 TO item10 (7). COUNT seven_c $=$ item11 TO item15 (7).

${ }^{*}$ Next, use these auxiliary variables to compute response style indices. ${ }^{*}$ ARS.

COMPU ars_a $=\left(3^{*}\right.$ seven_a $+2^{*}$ six_a + five_a $) / 15$. COMPU ars $\_b=\left(3^{*}\right.$ seven_b $+2^{*}$ six_b + five $\left.\_b\right) / 15$. COMPU ars_c $=\left(3^{*}\right.$ seven_c $+2^{*}$ six_c + five_c $) / 15$. EXE.

${ }^{*}$ DRS.

COMPU drs_a $=\left(3^{*}\right.$ one_a $+2^{*}$ two_a + three_a $) / 15$. COMPU drs_ $\mathrm{b}=\left(3^{*}\right.$ one $\mathrm{b} \mathrm{b}+2^{*}$ two_ $\mathrm{b}+$ three_ $\left.\mathrm{b}\right) / 15$. COMPU drs_c $=\left(3^{*}\right.$ one_c $+2^{*}$ two_c $c+$ three_c $) / 15$. EXE. ${ }^{*}$ ERS. COMPU ers_a $=($ one_a + seven_a $) / 15$. COMPU ers_b $=($ one_b + seven_b $) / 15$. COMPU ers_c $c=($ one_c $c+$ seven_c $) / 15$.

EXE.

${ }^{*}$ MRS.

COMPU mrs_a=four_a/ 15 . COMPU mrs_b=four_b/15. COMPU mrs_c=four_c $/ 15$. EXE.

\section{KEYWORDS:}

Cross-mode Surveys, Response Styles, Online Research, RIRSMACS 